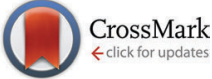

Cite this: Phys. Chem. Chem. Phys., 2016, 18, 14323

Received 13th February 2016, Accepted 19th April 2016

DOI: $10.1039 / \mathrm{c} 6 \mathrm{cp} 00995 f$

www.rsc.org/pccp

\section{Hydrogen dynamics in $\beta-\mathrm{Mg}\left(\mathrm{BH}_{4}\right)_{2}$ on the picosecond timescale}

\begin{abstract}
Luca Silvi, ${ }^{a}$ Eva Röhm, ${ }^{b}$ Maximilian Fichtner, ${ }^{b}$ Winfried Petry ${ }^{a}$ and Wiebke Lohstroh ${ }^{a}$
A quasielastic neutron scattering study on $\beta-\mathrm{Mg}_{(}\left(\mathrm{BH}_{4}\right)_{2}$ has been performed to investigate the hydrogen dynamics on the picosecond time-scale. Both vibrational and rotational motions of the $\left[\mathrm{BH}_{4}\right]^{-}$tetrahedra contribute to the signal at low energy transfers. A comprehensive analysis of the elastic and quasielastic incoherent structure factors allowed the separation of different parts. Below $200 \mathrm{~K}$, vibrations and rotations (around the $C_{2}$ or $C_{3}$ symmetry axis of the $\left[\mathrm{BH}_{4}\right]^{-}$tetrahedra) are well separated. Above that temperature, a transition is observed in the vibrational part, and the spectral weight is shifted towards the quasielastic region. The dynamic transition is not accompanied by any structural phase change but we suggest that it is correlated with the anomalous thermal expansion that has been reported for $\beta-\mathrm{Mg}\left(\mathrm{BH}_{4}\right)_{2}$ [Filinchuk, et al., Chem. Mater., 2009, 21, 925].
\end{abstract}

\section{Introduction}

Complex borohydrides are compounds composed of a metal cation (e.g. $\mathrm{Li}, \mathrm{Mg}, \mathrm{Ca}$, or $\mathrm{K}$ ) and a tetrahedral unit $\left[\mathrm{BH}_{4}\right]$. This material class has been the subject of intensive studies due to its promising hydrogen storage capacity. For example, magnesium borohydride $\left(\mathrm{Mg}\left(\mathrm{BH}_{4}\right)_{2}\right)$ is characterized by a gravimetric and volumetric storage density of $14.9 \mathrm{wt} \%$ and $90 \mathrm{~kg} \mathrm{~m}^{-3} \mathrm{H}_{2} ;{ }^{1,2}$ moreover, the material is interesting due to its overall reaction enthalpy $\Delta H$, and the reported values (for desorption to $\mathrm{MgH}_{2}$ ) are in the range of -40 to $-57 \mathrm{~kJ} \mathrm{~mol}^{-1} \mathrm{H}_{2}{ }^{3,4}$ which is suitable for storage applications. However, reaction kinetics is sluggish and $\mathrm{Mg}\left(\mathrm{BH}_{4}\right)_{2}$ decomposes only at $600 \mathrm{~K}$ in a multi-step process ${ }^{5,6}$ and its limited reversibility ${ }^{7}$ makes it unsuitable for practical applications.

Magnesium borohydride exists in various crystalline forms, as recently discovered and characterized by X-ray and neutron scattering techniques. ${ }^{8-10}$ Among the observed polymorphs of $\operatorname{Mg}\left(\mathrm{BH}_{4}\right)_{2}$, namely $\alpha, \beta, \gamma$ and $\delta$-phases, the low-temperature (LT) $\alpha$-phase and the high-temperature (HT) $\beta$-phase are the most commonly investigated for hydrogen storage applications; ${ }^{7}$ the crystal structures are shown in Fig. 1.

The structure of $\alpha-\mathrm{Mg}\left(\mathrm{BH}_{4}\right)_{2}$ has been identified by X-ray and neutron scattering techniques to be a hexagonal lattice with $P 6{ }_{1} 22$ space group symmetry and a unit cell volume of $3434 \AA^{3} .{ }^{11}$ The $\alpha$-polymorph is stable up to $490 \mathrm{~K}$ and, at higher temperatures, it transforms irreversibly into the $\beta$-phase. ${ }^{12,13}$ The latter has an

\footnotetext{
${ }^{a}$ Heinz Maier-Leibnitz Zentrum (MLZ) und Physik Department E13, Technische Universität München, Lichtenbergstr. 1, 85748, Garching, Germany.

E-mail: luca.silvi@frm2.tum.de

${ }^{b}$ Karlsruher Institut für Technology, Institut für Nanotechnologie, P. O. Box 3640, D-76021 Karlsruhe, Germany
}

orthorhombic Fddd crystal structure symmetry with a larger unit cell volume of $7543 \AA^{3}$ and it is metastable when cooled down to room temperature (and below). In comparison to the $\beta$-phase, the $\alpha$-phase is less dense (3\%) and it contains some unoccupied voids $\left(37 \AA^{3}\right)$ along the $(00 z)$ direction which are not present in the $\beta$-polymorph.

The hexagonal $P 6_{1} 22$ structure is characterized by six nonequivalent $\left[\mathrm{BH}_{4}\right]^{-}$positions, whereas in the orthorhombic $\mathrm{Fddd}$ structure the non-equivalent positions decrease to five. In both phases, $\mathrm{Mg}$ atoms are surrounded by four $\left[\mathrm{BH}_{4}\right]$ tetrahedra leading to a strongly distorted tetrahedral environment, and, at the local level, the $\left[\mathrm{BH}_{4}\right]$ groups are facing the $\mathrm{Mg}$ atoms via two opposite edges of the tetrahedra, leading to an almost linear configuration $\mathrm{Mg}-\mathrm{B}-\mathrm{Mg}$ and a bidentate orientation of $\mathrm{Mg}$ with respect to the bridging hydrogens ${ }^{11,12}$ (Fig. 2).

The decomposition of $\beta-\mathrm{Mg}\left(\mathrm{BH}_{4}\right)_{2}$ has been studied extensively for hydrogen storage applications and various pathways have been observed both experimentally and theoretically, depending on the applied hydrogen pressure and temperature conditions. ${ }^{6,14}$ In order to improve the slow kinetics, $\mathrm{Mg}\left(\mathrm{BH}_{4}\right)_{2}$ has been mixed with additives, mainly metal salts such as $\mathrm{CaF}_{2}, \mathrm{ZnF}_{2}$ and $\mathrm{TiF}_{3},{ }^{15} \mathrm{ScCl}_{3}{ }^{16}$ or $\mathrm{NbF}_{5} \cdot{ }^{17}$ Moreover, the mixtures with other borohydrides (e.g. $\left.\mathrm{Li}\left(\mathrm{BH}_{4}\right)\right)$ have been investigated in detail. ${ }^{18}$ Noteworthily, in the latter case, it is possible to reverse the structural phase transition from the $\beta$ - to $\alpha$-phase during cooling of the sample, although the mechanism is not entirely understood.

Despite the numerous studies, a full understanding of the ground state structures, hydrogen bonding and gas release is still missing. Extensive theoretical calculations show that the ground state is energetically almost degenerated and a variety of different structures that are very close in energy have been proposed, among them the experimentally found $\alpha$-phase. ${ }^{19} \mathrm{~A}$ recent work ${ }^{20}$ 

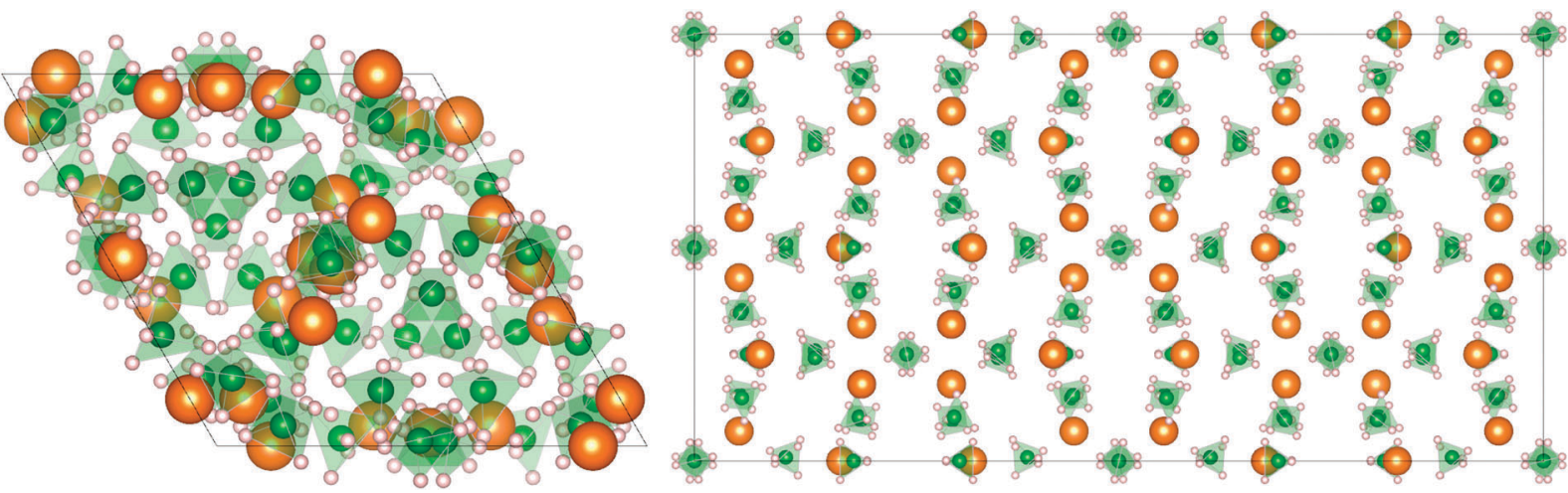

Fig. 1 Crystal structure of $\alpha$ - (left) and $\left.\beta-\mathrm{Mg}_{(\mathrm{BH}}\right)_{2}$ (right) along the $c$ axis: magnesium atoms are represented by orange spheres, boron by green and hydrogen by gray spheres. In both phases, $\mathrm{Mg}$ atoms are surrounded by four $\left[\mathrm{BH}_{4}\right]$ units in a strongly deformed tetrahedral environment. ${ }^{11}$

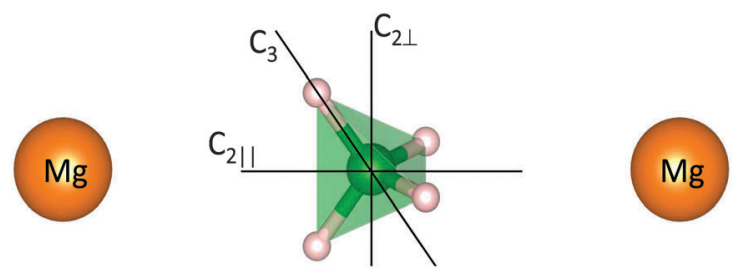

Fig. 2 Idealized configuration of the $\left[\mathrm{BH}_{4}\right]$ units in the $\mathrm{Mg}$ environment. The $\left[\mathrm{BH}_{4}\right]$ unit lies almost in the line between two $\mathrm{Mg}$ atoms in a linear configuration. The three rotation axes are shown: the 3 -fold $120^{\circ}$ rotation axis $C_{3}$, and the 2 -fold $180^{\circ}$ rotation axes $\left(C_{2 \|}\right.$ and $C_{2 \perp}$ ). (large orange sphere) magnesium, (green spheres) boron, and (small grey spheres) hydrogen.

showed that, including van der Waals interactions, the lowest energy structure is the experimentally observed $\alpha$-phase. Conversely, Caputo et al., ${ }^{21}$ using a computational methodology combining cluster optimization, simulated annealing based crystal structure prediction, periodic DFT total energy calculations and symmetry group relations analysis, found other crystal structures with lower energies. Still, the question about theoretically predicted and experimentally observed structures is open. The $\beta$-phase is energetically less favourable than the $\alpha$ phase, as shown by DFT calculations, ${ }^{2-24}$ but the origin of the non-reversibility of the structural phase transition is not clear yet. For the alkaline borohydrides $\mathrm{LiBH}_{4},{ }^{25} \mathrm{NaBH}_{4}{ }^{26}$ and $\mathrm{KBH}_{4},{ }^{27}$ it has been observed from quasielastic neutron scattering (QENS) experiments that the driving force for the structural phase transition is an order-disorder transition of the $\left[\mathrm{BH}_{4}\right]$ units, whereas for $\mathrm{Ca}\left(\mathrm{BH}_{4}\right)_{2}$ it was suggested that the $\left[\mathrm{BH}_{4}\right]$ librations drive the structural phase transition. ${ }^{28}$ Apparently, the dynamics of the $\left[\mathrm{BH}_{4}\right]$ units plays a crucial role in understanding the thermal properties of alkaline and earth alkaline borohydrides.

For $\mathrm{Mg}\left(\mathrm{BH}_{4}\right)_{2}$, nuclear magnetic resonance (NMR) experiments ${ }^{29,30}$ revealed a complex reorientational dynamic involving at least three jump processes in $\alpha-\mathrm{Mg}\left(\mathrm{BH}_{4}\right)_{2}$, and a variety of thermally activated processes in $\beta-\mathrm{Mg}\left(\mathrm{BH}_{4}\right)_{2}$ with a distribution of activation energies. QENS experiments performed on $\beta-\mathrm{Mg}\left(\mathrm{BH}_{4}\right)_{2}{ }^{31}$ showed the reorientation of the $\left[\mathrm{BH}_{4}\right]$ tetrahedra on two different characteristic timescales, using backscattering and indirect geometry time-of-flight spectroscopy. From the elastic incoherent structure factor (EISF) (which correlates with the spatial distribution of the hydrogens in the long time average), it was suggested that the $\left[\mathrm{BH}_{4}\right]$ units exhibit hindered rotations around the 2-fold $C_{2}$ and 3-fold $C_{3}$ symmetry axis of the $\left[\mathrm{BH}_{4}\right]$ units, respectively (see Fig. 2) on a timescale of hundreds of picoseconds.

The aim of the present work is to investigate the hydrogen dynamics in an extended dynamic range, i.e. to address the picosecond timescale and extend the momentum transfer $(Q)$ to larger values to shed further light on the hydrogen dynamics in $\beta-\mathrm{Mg}\left(\mathrm{BH}_{4}\right)_{2}$. In particular, the evaluation of the EISF and the quasielastic incoherent structure factor (QISF) at large $Q$ values enables the discrimination of different reorientational geometries. Moreover, QENS experiments might reveal a significant contribution of vibrational motions in the experimentally probed time-window.

\section{Experimental}

Quasielastic neutron scattering (QENS) has been performed on $\beta-\mathrm{Mg}\left(\mathrm{BH}_{4}\right)_{2}$. The material has been prepared as described in previous work. ${ }^{32}$ The high neutron absorption cross-section of natural boron was avoided using isotope enriched ${ }^{11} \mathrm{~B}$. For the measurements, approx. $200 \mathrm{mg}$ of $\beta-\mathrm{Mg}\left({ }^{11} \mathrm{BH}_{4}\right)_{2}$ was mounted into an Aluminium flat cell, yielding a neutron transmission of $\sim 90 \%$. Quasielastic neutron scattering experiments have been performed using a cold time-of-flight spectrometer TOFTOF ${ }^{33,34}$ at the Heinz Maier-Leibnitz Zentrum (MLZ) in Munich, Germany. Two different wavelengths of incoming neutrons were selected: the first at $\lambda_{1}=2.5 \AA$ yielding an energy resolution of $450 \mu \mathrm{eV}$ (FWHM) of the elastic line and an accessible elastic momentum transfer $Q$ from 0.5 to $4.2 \AA^{-1}$, and the second at $\lambda_{2}=6 \AA$, with an energy resolution of $48 \mu \mathrm{eV}$ (FWHM) and an elastic momentum transfer from 0.2 to $2 \AA^{-1}$. The sample was mounted at $135^{\circ}$ with respect to the incoming neutron beam and the measurements were performed at temperatures ranging from 11 to $500 \mathrm{~K}$. The raw time-of-flight data were converted to the scattering function $S(Q, \omega)$ by the following procedure: the data were normalized to the incoming neutron flux and the empty container signal was 
subtracted taking into account self-shielding and self-absorption factors. After normalizing to a vanadium standard measurement, the energy transfer was calculated and a correction of energy dependent detector efficiency was performed. The momentum transfer $Q$ was calculated and the spectra have been grouped into bins of equal $\Delta Q$. For the analysis, detector angles of the aluminium Bragg peaks arising from the sample environment were excluded.

\section{Results and discussion}

The results of QENS measurements are shown in Fig. 3 and 4 for the two wavelengths and a chosen $Q$-value. For both instrument settings, the sample has been cooled down to $11 \mathrm{~K}$. At this temperature, the measured width of the elastic peak at zero

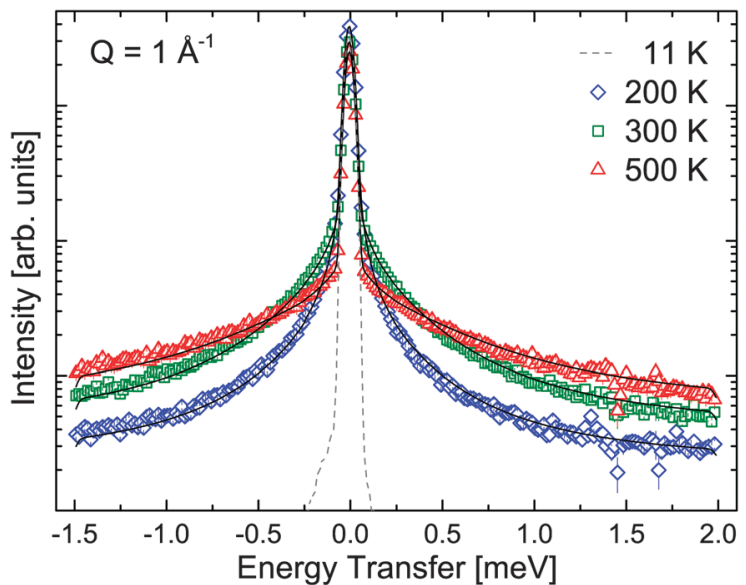

Fig. $3 S(Q, \omega)$ at $6 \AA$ of $\beta-M g\left(B H_{4}\right)_{2}$ at $11,200,300$ and $500 \mathrm{~K}$. The black solid lines represent the fit of the data according to eqn (11). (gray dashed line) $11 \mathrm{~K}$ (resolution), (blue rhombus) $200 \mathrm{~K}$, (green square) $300 \mathrm{~K}$, and (red triangle) $500 \mathrm{~K}$. The vertical error bars denote $\pm 1 \sigma$.

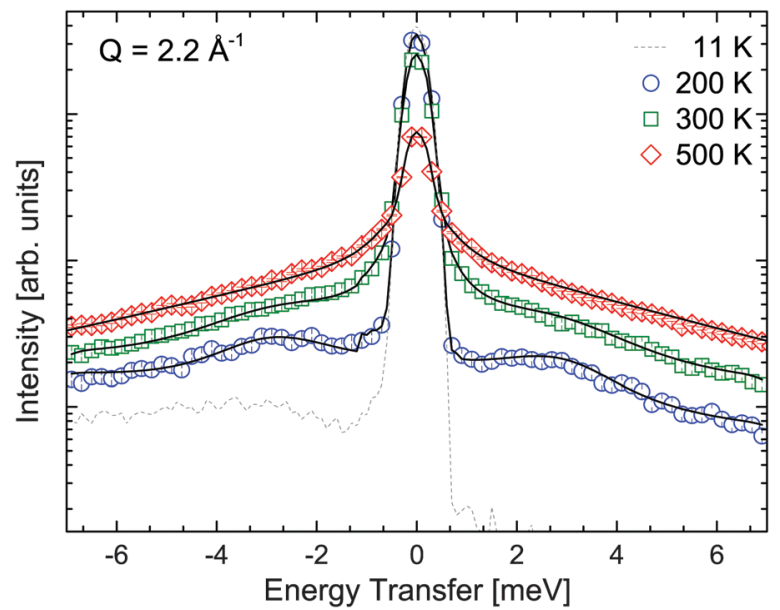

Fig. $4 S(Q, \omega)$ of $\beta-M g\left(B H_{4}\right)_{2}$, measured at $2.5 \AA$ and at $11,100,200$ and $500 \mathrm{~K}$. The black solid lines represent the fit of the data according to eqn (11) and (14). (gray line) $11 \mathrm{~K}$ (resolution), (blue circle) $100 \mathrm{~K}$, (green square) $200 \mathrm{~K}$, and (red triangle) $500 \mathrm{~K}$. Datasets measured at 300 and $400 \mathrm{~K}$ have been omitted for clarity. The vertical error bars denote $\pm 1 \sigma$. energy transfer reflects the instrumental resolution function. With increasing temperature, a quasielastic broadening is observed, and its intensity increases with increasing temperature. Note that the two incoming wavelengths $\lambda$ probe different characteristic timescales and hence the observed quasielastic signals could be caused by different underlying motions. Besides the quasielastic broadening, the data probing the shorter timescales $(\lambda=2.5 \AA)$ show distinct low energy (vibrational) inelastic peaks at $\Delta E= \pm 3.2 \mathrm{meV}$ for temperatures below $300 \mathrm{~K}$. The energy transfer of this excitation is constant for all $Q$. At $300 \mathrm{~K}$ and above, the quasielastic signal becomes very strong and the inelastic excitation cannot be resolved in the spectra.

The first attempt to separate the vibrational and quasielastic signals was carried out by following the procedure proposed by Cusack et $a l .{ }^{35}$ In the harmonic approximation, the vibrational frequency distribution is temperature independent, and the Debye-Waller factor and the Bose occupation number relate the data taken at different temperatures. Outside the QENS region, the relation with low temperature data and high temperature data is then given by:

$$
\frac{S(Q, \omega, T)}{S\left(Q, \omega, T_{0}\right)} \frac{\mathrm{e}^{-Q^{2} U_{\mathrm{vib}}\left(T_{0}\right)}}{\mathrm{e}^{-Q^{2} U_{\mathrm{vib}}(T)}}=\frac{n(\omega, T)}{n\left(\omega, T_{0}\right)} \approx \frac{T}{T_{0}}\left[1+\frac{\hbar \omega}{2 k_{\mathrm{B}}}\left(\frac{1}{T_{0}}-\frac{1}{T}\right)\right] .
$$

In the above equation, $T_{0}$ is the reference temperature where no quasielastic intensity is detected, in this case $T_{0}=100 \mathrm{~K}$. A plot of the left hand side of eqn (1) is shown in Fig. 5, together with the linear dependence of the right hand side of eqn (1).

The vibrational displacement $U_{\text {vib }}$ in eqn (1) is temperature dependent and the best agreement with the data is obtained for $U_{\text {vib }}=0.0001 \times T[\mathrm{~K}] \AA^{2}$. Clearly at $200 \mathrm{~K}$ the quasielastic signal falls to zero beyond $\sim 2 \mathrm{meV}$. Above $\sim 2 \mathrm{meV}$, the signal is described to a good approximation by the harmonic temperature scaling, whereas above $300 \mathrm{~K}$, the agreement with the approximation is only found for energies above $\sim 8 \mathrm{meV}$. This is an

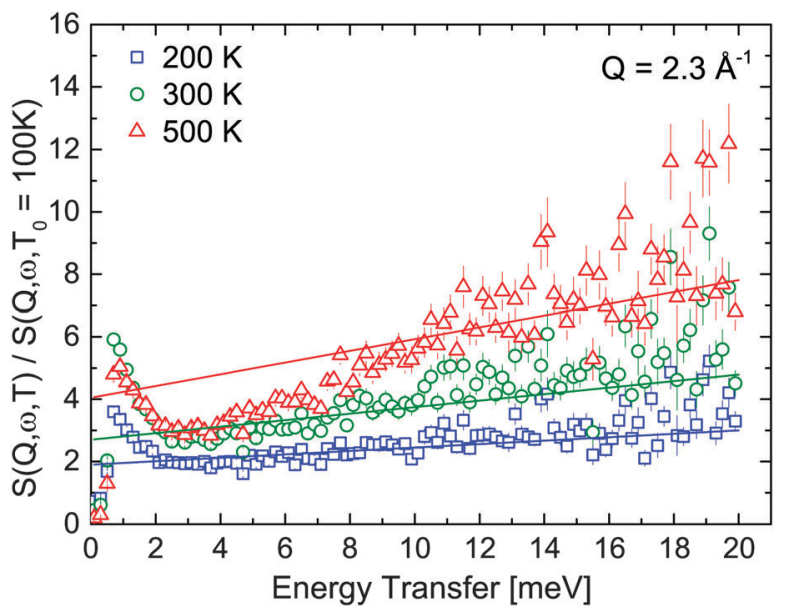

Fig. 5 Ratio between high temperature data and low temperature data ( $T=100 \mathrm{~K}$ ) at $\lambda=2.5 \AA$ : (blue squares) $200 \mathrm{~K}$, (green circles) $300 \mathrm{~K}$, (red triangles) $500 \mathrm{~K}$. Data at $400 \mathrm{~K}$ have been omitted for clarity. The line represents the right hand-side of eqn (1), using $U_{\text {vib }}=0.0001 \times T[K] \AA^{2}$. The vertical error bars denote $\pm 1 \sigma$. 


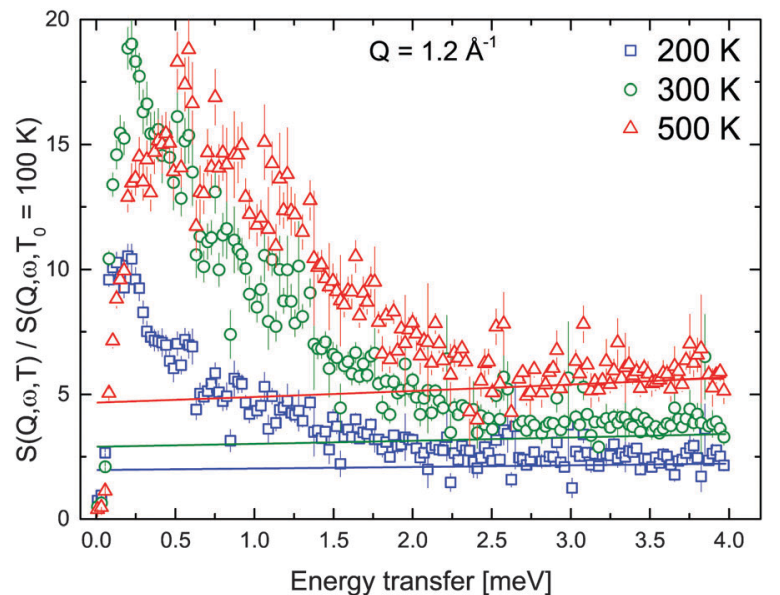

Fig. 6 Ratio between high temperature data and low temperature data ( $T=100 \mathrm{~K}$ ) at $\lambda=6 \AA$ : (blue squares) $200 \mathrm{~K}$, (green circles) $300 \mathrm{~K}$, (red triangles) $500 \mathrm{~K}$. Data at $400 \mathrm{~K}$ have been omitted for clarity. The line represents the right hand-side of eqn (1), using $U_{\text {vib }}=0.0001 \times T[K] \AA^{2}$. The vertical error bars denote $\pm 1 \sigma$.

indication of a transition in the vibrational modes that affects the quasielastic region. A clear separation of these two contributions is not possible and the vibrational part cannot be subtracted for analysis of the quasielastic data. Therefore, a more complex approach was chosen, as outlined below.

The same analysis for the $6 \AA$ data is shown in Fig. 6. Above $\sim 2 \mathrm{meV}$ the quasielastic signal falls off, showing a good agreement with the right hand side of eqn (1) and hence in this case, the vibrational part could be treated as flat background contribution.

\subsection{Data analysis}

Due to the large hydrogen incoherent scattering cross-section, the experiments almost exclusively probe hydrogen dynamics. In the incoherent approximation, the scattering function $S(Q, \omega)$ can be written as:

$$
S(Q, \omega)=S_{\text {trans }}(Q, \omega) \otimes S_{\text {rot }}(Q, \omega) \otimes S_{\text {vib }}(Q, \omega),
$$

where $S_{\text {trans }}, S_{\text {rot }}$ and $S_{\text {vib }}$ are the scattering functions of translational, rotational and vibrational diffusion, and $\otimes$ denotes the convolution operator. The previous equation is valid for independent motions. The measured intensity is related to the scattering function via a convolution with the instrument resolution function $\operatorname{Res}(Q, \omega)$. For the experimental data shown above, we suppose a combination of rotational and vibrational reorientations (i.e. no translational diffusion). In this case, the scattering function can be rewritten as:

$$
I_{\text {meas }} \propto\left[S_{\text {rot }}(Q, \omega) \otimes S_{\text {vib }}(Q, \omega)\right] \otimes \operatorname{Res}(Q, \omega)+b(Q) .
$$

The term $b(Q)$ is the background originating from motions that are much faster than the timescale set by the instrument resolution.

The fraction of elastically scattered neutrons

$$
\operatorname{EISF}(Q)=\frac{I_{\mathrm{el}}(Q)}{I_{\mathrm{el}}(Q)+I_{\text {inel }}(Q)}
$$

is called the elastic incoherent structure factor (EISF) which can be evaluated directly from the measured elastic, inelastic and quasielastic integrated intensities, independent of any specific model. Another quantity of interest in this work is the Quasielastic Incoherent Structure Factor, namely QISF. It is related to the EISF, through the sum rule $\operatorname{EISF}(Q)+\operatorname{QISF}(Q)=1$, and it is calculated as the ratio of the inelastic (or quasielastic) intensity over the total integrated intensity. The EISF is the spatial Fourier transform of the probability function to find a hydrogen atom at a given position, and hence its functional dependence on the momentum transfer $Q$ reflects the spatial distribution of the hydrogen atoms in the long time average. ${ }^{36}$ For specific motions, the EISF can be calculated and the comparison with experimental data is used to discriminate between different reorientation mechanisms. As a consequence, a large part of the momentum transfer should be explored.

The experimental $S(Q, \omega)$ has been analysed as outlined in Appendix A, and from that the EISF, QISF ${ }_{\mathrm{QE}}$ and QISF $_{\text {inel }}$ have been derived for each dataset. For the three quantities it is:

$$
\begin{gathered}
\operatorname{EISF}=B(Q)\left[p+(1-p) A_{0}(Q)\right] \\
\operatorname{QISF}_{\mathrm{QE}}=B(Q)(1-p)\left[1-A_{0}(Q)\right] \\
\operatorname{QISF}_{\text {inel }}=1-B(Q),
\end{gathered}
$$

with $B(Q)$ being the Debye-Waller factor, $A_{0}$ being the EISF for a jump rotational diffusion around the symmetry axes $C_{2}$ or $C_{3}$, and $p$ being the fraction of $\left[\mathrm{BH}_{4}\right]$ units not undergoing rotational dynamics.

$$
\begin{gathered}
A_{0}(Q)_{C_{2 / 3}}=\frac{1}{2}\left[1+j_{0}\left(\frac{2 \sqrt{2}}{\sqrt{3}} Q d_{\mathrm{B}-\mathrm{H}}\right)\right], \\
B(Q)=\exp \left(-\frac{Q^{2}\left\langle u^{2}\right\rangle}{3}\right) .
\end{gathered}
$$

Details of the scattering functions, and quantities derived (i.e. EISFs and QISFs) and used in the next sections are given in Appendix A. The data analysis will be split into two parts: data taken at longer timescales and data at shorter timescales.

\subsection{Data analysis for $\lambda=6 \AA$}

For the analysis of the measurements performed at $\lambda=6 \AA$, the spectra have been fitted up to $2 \mathrm{meV}$. The vibrational part is then described by a flat background (see beginning of this section) and in all the fit functions the correct dependence of the detailed balance factor has been taken into account. For $T \geq 200 \mathrm{~K}$, the data show a clear quasielastic component and two Lorentzians in eqn (11) are necessary to describe the experimental points: the first one with a half width half maximum (HWHM) $\Gamma_{1}<0.1 \mathrm{meV}$ and a second, broader one with a $\Gamma_{2}>0.3 \mathrm{meV}$. For both Lorentzians, $\Gamma_{1}$ and $\Gamma_{2}$ do not show any $Q$-dispersion, however, the widths are increasing with temperature, indicating thermally activated motions localized in space. ${ }^{37}$ A summary of the results for $\Gamma_{1}$ and $\Gamma_{2}$ can be found in Table 1. 
Table 1 The HWHM $\Gamma_{1}$ and $\Gamma_{2}$ used for describing the data at $6 \AA$ and at different temperatures. In addition, $\delta(\omega)$ is used for fitting the elastic contributions at all temperatures. Data at $100 \mathrm{~K}$ show no quasielastic signals

\begin{tabular}{lcl}
\hline$T[\mathrm{~K}]$ & $\Gamma_{1}[\mathrm{meV}]$ & $\Gamma_{2}[\mathrm{meV}]$ \\
\hline 100 & - & - \\
200 & $0.033 \pm 0.002$ & $0.33 \pm 0.05$ \\
300 & $0.073 \pm 0.011$ & $0.35 \pm 0.04$ \\
400 & $0.08 \pm 0.008$ & $0.50 \pm 0.06$ \\
500 & $0.11 \pm 0.02$ & $0.55 \pm 0.08$
\end{tabular}

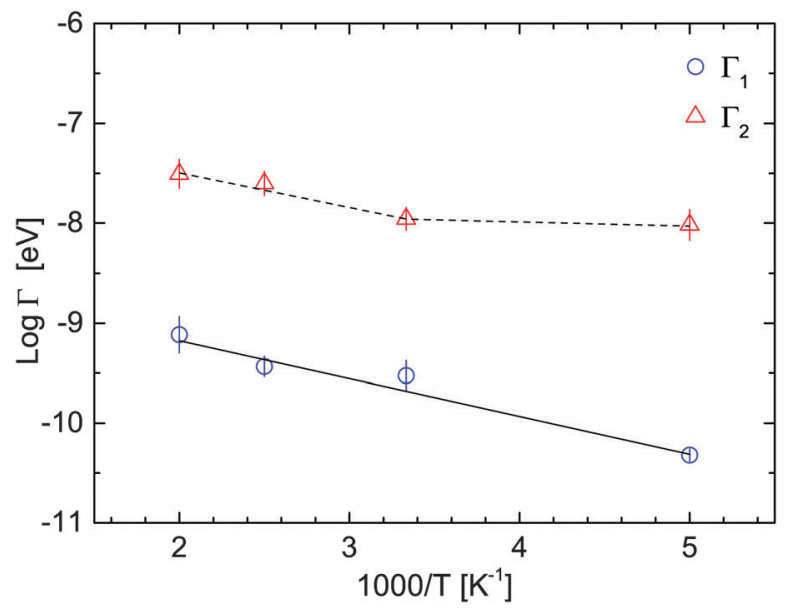

Fig. 7 Linewidths $\Gamma_{1}$ (blue circles) and $\Gamma_{2}$ (red triangles) on a logarithmic scale measured at $6 \AA$ as a function of temperature. $\Gamma_{1}$ values are fitted with the Arrhenius function $\Gamma=\Gamma_{0} \exp \left(-E_{\mathrm{a}} / k_{\mathrm{B}} T\right)$. The dashed line (guide to the eye) shows different slopes, indicating a transition phase of the faster motion $\Gamma_{2}$ (see the text for explanations). The vertical error bars denote $\pm 1 \sigma$.

Fig. 7 shows $\Gamma_{1}$ and $\Gamma_{2}$ as a function of the temperature (on a logarithmic scale), together with the fit of the Arrhenius expression:

$$
\Gamma=\Gamma_{0} \exp \left(-\frac{E_{\mathrm{a}}}{k_{\mathrm{B}} T}\right)
$$

where $\Gamma_{0}$ is a pre-factor, $E_{\mathrm{a}}$ is the apparent activation energy, $T$ is the temperature and $k_{\mathrm{B}}$ is the Boltzmann constant. For the slower process $\Gamma_{1}$, the characteristic timescale is $5-20 \mathrm{ps}$ and the apparent activation energy was determined to be $E_{\mathrm{a}}=32 \pm 3$ $\mathrm{meV}$ and $\Gamma_{0}=0.21 \mathrm{meV}$. As seen in Fig. 7, there is an indication of a transition in the functional dependence of $\Gamma_{2}$ at around $300 \mathrm{~K}$. The dashed line shown is a guide to the eye, only. For this reason we do not attribute a single Arrhenius activation energy over the entire temperature range.

The elastic incoherent structure factor (EISF) has been calculated using eqn (4), and the results are plotted in Fig. 8. Also shown are the theoretically expected $Q$-dependence of the EISF for rotations around the $C_{2} / C_{3}$ axis and the tumbling motion. At $300 \mathrm{~K}$ and above, the EISF is independent of temperature, indicating that the spatial distribution of the hydrogen atoms does not change in the probed time- and space window, however the increasing $\Gamma_{1,2}$ confirms that reorientation processes become faster with increasing temperature. The functional $Q$-dependence will

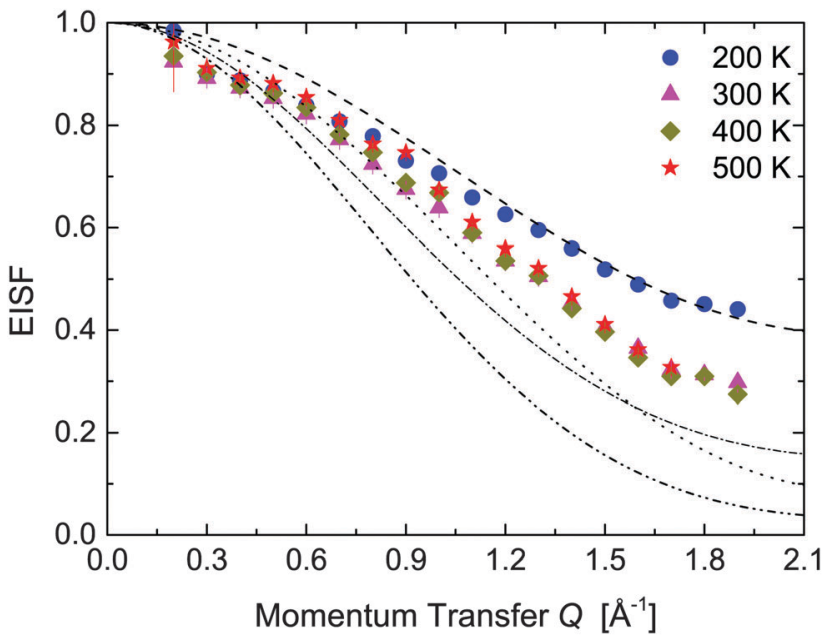

Fig. 8 Measured EISFs at $6 \AA$ : (blue circles) $200 \mathrm{~K}$, (magenta triangles) $300 \mathrm{~K}$, (yellow rhombus) $400 \mathrm{~K}$, and (red stars) $500 \mathrm{~K}$. The dashed line represents the rotational diffusion model for $C_{2 / 3}$ rotations (eqn (8)), whereas the dotted line represents the tumbling motions, according to eqn (17). The dash-dot line is a combination of the $C_{2}$ and $C_{3}$ rotations, and the dash-dot-dot line is a combination of $C_{2 / 3}$ rotation with tumbling motions (see the text for details). The vertical error bars denote $\pm 1 \sigma$.

be discussed later in the paper, in connection with the results obtained for the $2.5 \AA$ A-measurements.

\subsection{Data analysis for $\lambda=2.5 \AA$}

Analysis of the data obtained at $2.5 \AA$ (see Fig. 4) is more complex due to the observed distinct inelastic contribution close to the quasielastic region. These excitations are occurring around $\sim \pm 3.2 \mathrm{meV}$, and they have been also reported in ref. 38, and were tentatively ascribed to lattice phonons. The spectra have been fitted using the functions listed in Table 2 to represent the elastic, quasielastic and vibrational contributions, and the results of the fits are shown in Fig. 9 and 10. In the instrumental resolution function measured at $11 \mathrm{~K}$ (see Fig. 4), the inelastic excitation appears in the energy-loss side of the neutron and therefore only the central peak has been used as a resolution $\operatorname{Res}(Q, \omega)$ in eqn (3).

At 100 and $200 \mathrm{~K}$, data show clearly inelastic peaks close, but still separated from the quasielastic region. The vibrational part is modelled using a damped harmonic oscillator (DHO, with a frequency $\omega_{q}$, eqn (14)) and the jump rotational part according to eqn (11). The starting values for the jump rotational part are

Table 2 The fit functions used for describing data at $\lambda=2.5 \AA$ at different temperatures: a $\delta$-function is used for fitting the elastic contribution, a $\mathrm{DHO}$ is used to model the inelastic peaks (eqn (14)), and a background is used to model fast vibrational dynamics outside the set timescale; $L_{2}$ and $L_{3}$ represent the quasielastic contribution and the over-damped harmonic oscillator, respectively (see the text for details)

\begin{tabular}{ll}
\hline$T[\mathrm{~K}]$ & Fit functions \\
\hline 100 & $\delta+\mathrm{DHO}+$ background \\
200 & $\delta+L_{2}+\mathrm{DHO}+$ background \\
$>300$ & $\delta+L_{2}+L_{3}$
\end{tabular}




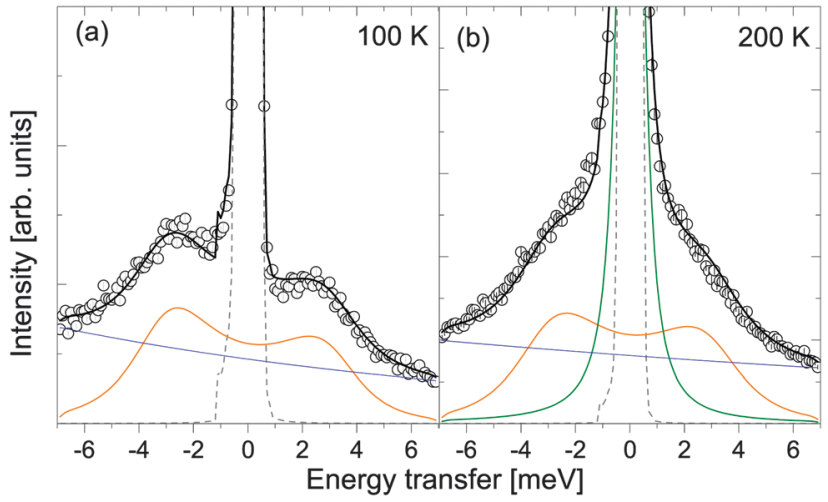

Fig. $9 S(Q, \omega)$ of $\beta-M g\left(B_{4}\right)_{2}$ measured at $Q=2.1 \AA^{-1}$ at 100 (a) and $200 \mathrm{~K}$ (b). Solid black lines represent the fit. In (a) data are successfully described by a DHO (orange) and a background (blue) that takes into account faster vibrations. In (b) data are described by a $\mathrm{DHO}$, a single Lorentzian (dark green) and a background. The vertical error bars denote $\pm 1 \sigma$.

taken from the $6 \AA$ data $\left(\Gamma_{2}\right)$, and the values found are in agreement with the $6 \AA$ data with $10 \%$ variation.

At temperature $T \geq 300 \mathrm{~K}$, data are not showing any visible inelastic peaks. At this temperature we presume an over-damped DHO which is indistinguishable from a Lorentzian shape function in the measured energy transfer range. Therefore, the inelastic contribution had been described by a Lorentzian $L_{3}$ (characterised by $\Gamma_{3}$ (see Fig. 10 and 11)).

$\omega_{q}$ represents the characteristic frequency of individual $\left[\mathrm{BH}_{4}\right]$ units, since we probe the incoherent scattering crosssection. Apparently, the lattice potential of all five different $\left[\mathrm{BH}_{4}\right]$ sites is similar, yielding distinct peaks. Inelastic neutron scattering (INS) measurements on $\beta-\mathrm{Mg}\left(\mathrm{BH}_{4}\right)_{2}{ }^{38}$ show a complex vibrational structure extending over a wide range of energies. A structured low-energy band (between 3 and $38 \mathrm{meV}$ ) has been reported and it was tentatively assigned to lattice phonons. The inelastic peaks detected in this work around $\pm 3.2 \mathrm{meV}$ are in agreement with this previous study, thus confirming a

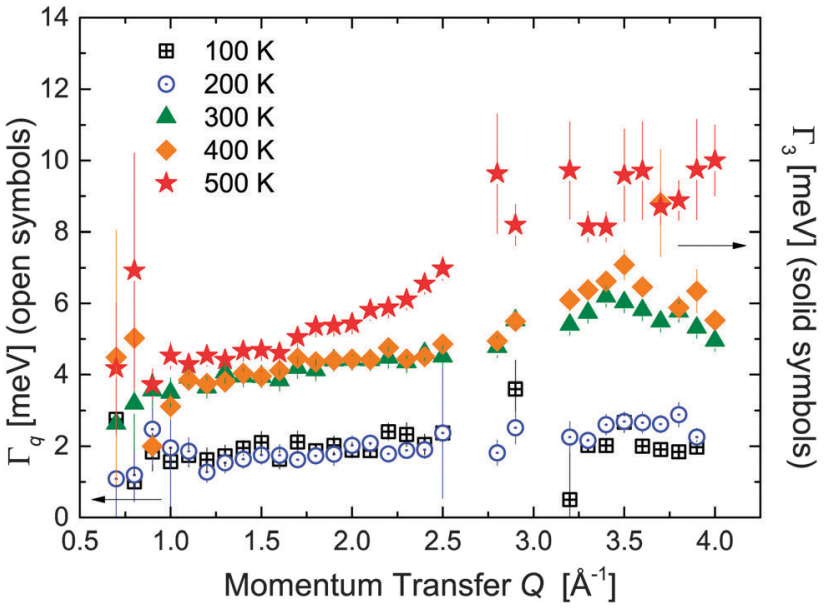

Fig. 11 Damping $\Gamma_{q}$ and HWHM $\Gamma_{3}$ at different temperatures. In the left $y$-axis: (open black squares) $100 \mathrm{~K}$ and (open blue circles) $200 \mathrm{~K}$. In the right $y$-axis: (green triangles) $300 \mathrm{~K}$, (yellow rhombus) $400 \mathrm{~K}$, and (red stars) $500 \mathrm{~K}$. The vertical error bars denote $\pm 1 \sigma$.

superposition of vibrational and quasielastic signals at low energy transfers. At higher temperatures, the vibrational diffusion becomes over-damped and is described in our analysis by $L_{3}$. Vibrational and rotational reorientations are occurring on similar timescales: the characteristic frequency extracted for vibration is $\nu_{\mathrm{vib}} \sim 0.8 \mathrm{ps}^{-1}$, which is in the same order of magnitude as rotational frequency $\tau_{\text {rot }}{ }^{-1}$ from $\Gamma_{2}$.

The EISF, QISF QE $_{\text {and }}$ QISF $_{\text {inel }}$ are calculated from the elastic, quasielastic and inelastic intensities, normalized to the total intensity. The results are summarized in Fig. 12. With increasing temperature, the fraction of elastically scattered neutrons (EISF) decreases, but no oscillating behaviour characteristic for rotational reorientations around the $C_{2}$ or $C_{3}$ axis is observed. This is expected, since no separation of vibrational and rotational parts was done. The solid lines in Fig. 12 are a global fit (at each temperature) to eqn (5)-(7) to extract the parameters $\left\langle u^{2}\right\rangle$,

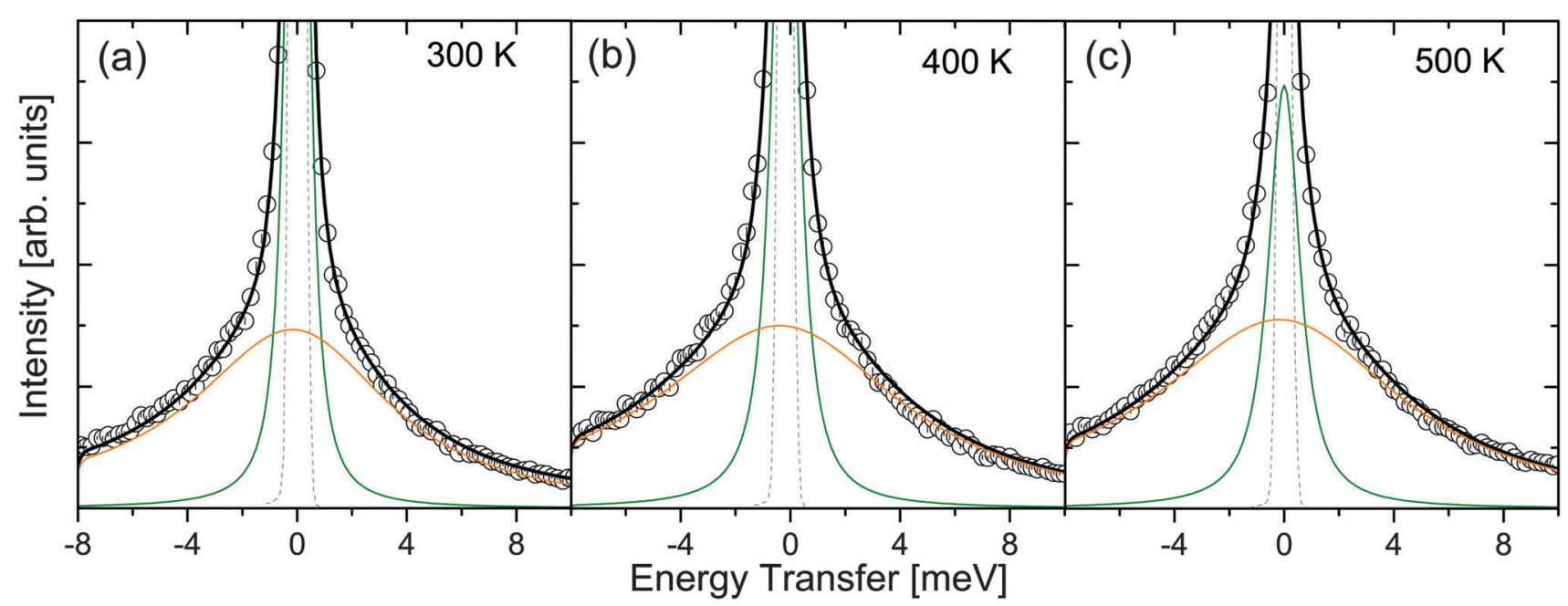

Fig. $10 S(Q, \omega)$ of $\left.\beta-\mathrm{Mg}_{(\mathrm{BH}}\right)_{2}$ measured at $Q=2.1 \AA^{-1}$ at $300 \mathrm{~K}(\mathrm{a}), 400 \mathrm{~K}$ (b), and $500 \mathrm{~K}$ (c). Solid black lines represent the fit. Data are described by two Lorentzians: $\Gamma_{2}$ (dark green) and $\Gamma_{3}$ (orange). The vertical error bars denote $\pm 1 \sigma$. 


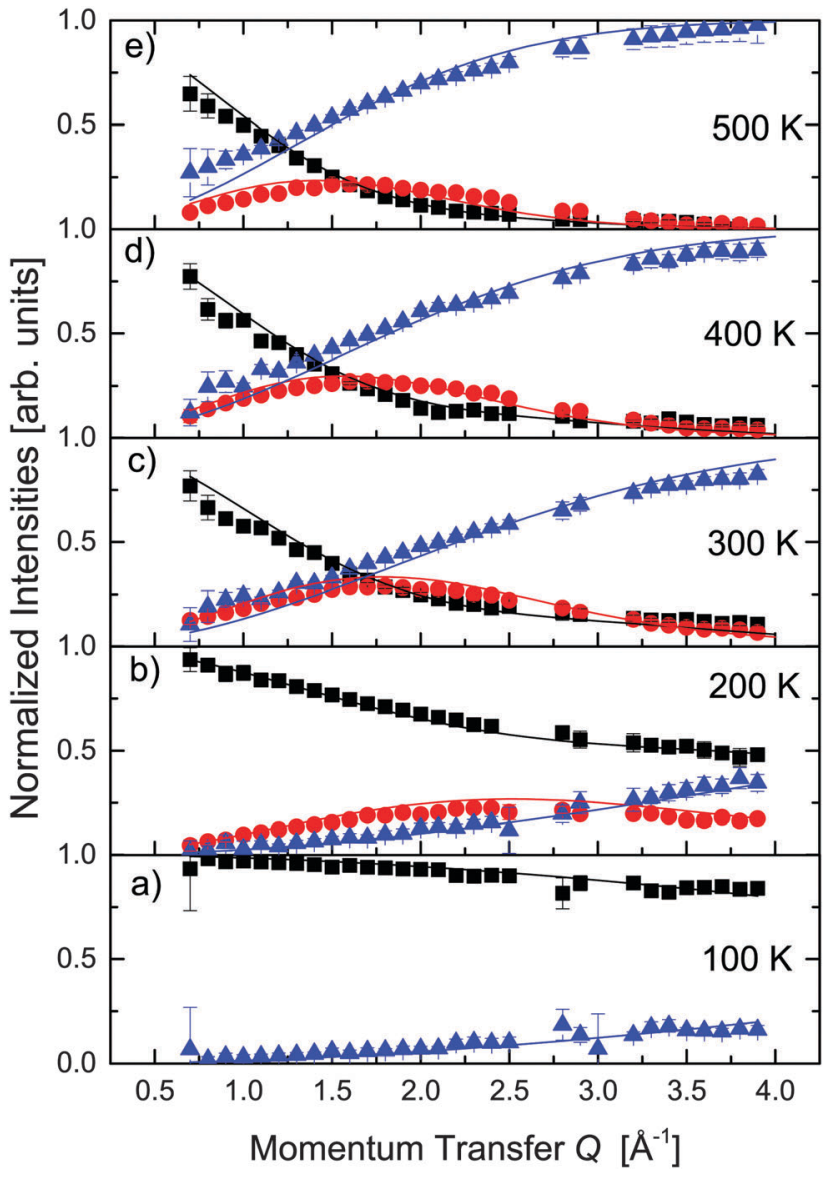

Fig. 12 EISF (black squares), QISF ${ }_{\text {inel }}$ (blue triangles), and QISF $_{L_{1}}$ (red circles) from $100 \mathrm{~K}$ to $500 \mathrm{~K}$. Solid lines represent the fit to the data according to eqn (5)-(7). In (a) $100 \mathrm{~K}$, (b) $200 \mathrm{~K}$, (c) $300 \mathrm{~K}$, (d) $400 \mathrm{~K}$ and (e) $500 \mathrm{~K}$. The vertical error bars denote $\pm 1 \sigma$.

$p$ and $d_{\mathrm{B}-\mathrm{H}}$. Due to the structureless behaviour at relatively high $Q$ of the EISF, this information cannot be obtained from the fraction of the elastically scattered neutrons alone, but the global fit procedure was necessary. For the data analysis, the $\mathrm{QE}$ components have been assumed to be described by $C_{2 / 3}$ reorientation (see eqn (8)), in agreement with low temperature data collected at $6 \AA$ and with the previously proposed reorientational mechanisms. ${ }^{31}$

The agreement with the experimental data is quite satisfactory confirming that the chosen approach for the analysis of the data allows us to extract the rotational and vibrational parts. The mean square displacement (MSD) $\left\langle u^{2}\right\rangle$ and $d_{\mathrm{B}-\mathrm{H}}$ are shown in Fig. 13 and 14.

\subsection{Discussion}

Previously, Blanchard et $a l .{ }^{31}$ reported three thermally activated processes in $\beta-\mathrm{Mg}\left(\mathrm{BH}_{4}\right)_{2}$ with activation energies: $39 \pm 0.5$, $76 \pm 5$ and $214 \pm 4 \mathrm{meV}$ from QENS data in the observation timescale of hundreds of picoseconds. In their work, the faster jump process with the lowest activation energy had been attributed to the rotation around the $C_{2 \|}$ axis (see Fig. 2), energetically more favourable because of the $\left[\mathrm{BH}_{4}\right]$ bidentate configuration with

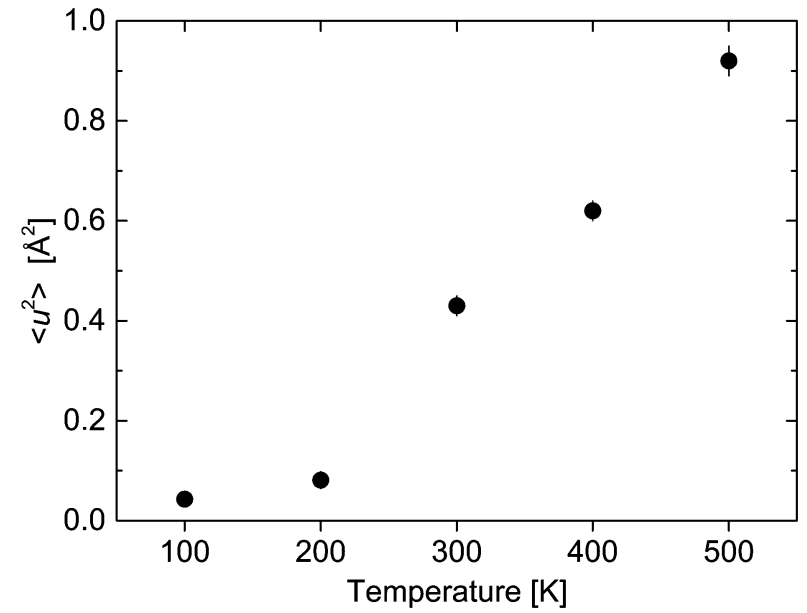

Fig. 13 Mean square displacement $\left\langle u^{2}\right\rangle$ as a function of temperature. The vertical error bars denote $\pm 1 \sigma$.

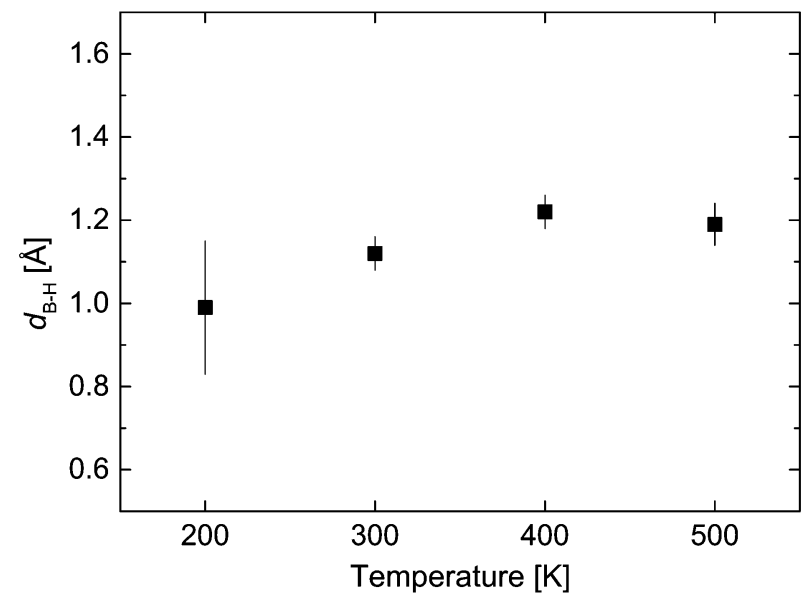

Fig. 14 Distance between boron and hydrogen atoms as a function of temperature. The vertical error bars denote $\pm 1 \sigma$.

respect to the $\mathrm{Mg}$ ions, although its weight was rather low $(15 \%$ of the QENS intensity). NMR measurements on $\beta-\mathrm{Mg}\left(\mathrm{BH}_{4}\right)_{2}{ }^{30}$ are not showing discrete activation energies, but a broad distribution, centred around $138 \pm 5 \mathrm{meV}$ with a distribution width of $\Delta E_{\mathrm{a}}=36 \pm 3 \mathrm{meV}$.

In this work, at longer timescales ( $6 \AA$-measurement), two reorientational processes with different time constants are detected. For the slower one, the activation energy has been calculated to be $E_{\mathrm{a}}=32 \pm 1 \mathrm{meV}$ and $\Gamma_{0}=0.22 \mathrm{meV}$, in agreement with previous QENS and DFT calculations. ${ }^{31}$ We tentatively assign a $C_{2 \|}$ reorientation, energetically more favorable with respect to $C_{3}$ rotations due to the bidentate configuration between $\mathrm{Mg}$ and $\mathrm{H}$ atoms. The other process is one order of magnitude faster $\left(\tau^{-1} \sim 0.5-1 \mathrm{ps}^{-1}\right)$, but it shows a discontinuity at around $300 \mathrm{~K}$ and therefore its activation energy has not been determined. $\Gamma_{2}$ is also of rotational origin, but its temperature dependence most likely reflects the transition in the vibrational part. The transition is also visible in the EISF, where, for temperatures above $200 \mathrm{~K}$, the deviation from simple reorientational motions 
(like $C_{2} / C_{3}$ rotations) becomes significant (see Fig. 8). Other reorientation processes (like tumbling motions (eqn (17))) or other combinations (convolution between $C_{2}$ and $C_{3}$ rotations, or between $C_{2}$ and tumbling motions) also do not describe the measured EISF obtained for the $6 \AA$ measurement.

Similarly, in the same temperature regime, the data measured at $2.5 \AA$ show the transition from an under-damped harmonic oscillator observed at 100 and $200 \mathrm{~K}$ to the over-damped regime above that temperature. The damping $\Gamma_{q}$ and the HWHM $\Gamma_{3}$ are plotted in Fig. 11. At $300 \mathrm{~K}$ and above, the vibrational inelastic scattering overlaps with the quasielastic region, and therefore the EISF reflects the merging of the two contributions.

The transition from the under-damped to the over-damped DHO is visible in the parameters extracted from the data analysis. The mean square displacement shows a step like increase from $200 \mathrm{~K}$ to $300 \mathrm{~K}$ and above (Fig. 13). The large values of $\left\langle u^{2}\right\rangle$ (Fig. 13) differ from the harmonic contribution $U_{\text {vib }}$ that has been estimated at the beginning of this section. $U_{\text {vib }}$ characterizes fast vibrations of single nuclei around their lattice position (Debye-Waller factor), whereas we attribute $\left\langle u^{2}\right\rangle$ to confined vibrational modes that originate from movements of the entire $\left[\mathrm{BH}_{4}\right]$ unit around their equilibrium position. Therefore, the MSD $\left\langle u^{2}\right\rangle$ describes in first approximation, the sum of contributions of localized atom vibrations and phonon band vibrations of the host lattice. For the lattice term, it is supposed that the hydrogen atoms follow the motions of the host lattice atoms. ${ }^{36}$

The rotational part, described by $L_{2}$, is detected at $200 \mathrm{~K}$ and above. The scattering intensity follows eqn (6), as shown in Fig. 12. At $200 \mathrm{~K}$, only a fraction $1-p$, with $p=0.47 \pm 0.08$, is undergoing reorientational motions, whereas at higher temperature, all $\left[\mathrm{BH}_{4}\right]$ are reorienting and therefore in eqn (5) and (6), $p$ is zero. The distances $d_{\mathrm{B}-\mathrm{H}}$ extracted are in the range of crystallographic values measured using $\mathrm{X}$-ray and neutron diffractions $^{11,12}$ (Fig. 14). From the analysis, we could assign the quasielastic rotational component to a $C_{2}$ reorientation mechanism.

From the analysis of the $2.5 \AA$ data, it became obvious that vibrational modes are contributing significantly to the observed intensities at low energy transfers. However, since the $6 \AA$ A-data are sensitive to longer timescales, it can be expected that $\left\langle u^{2}\right\rangle$ has different values. Similarly, we suppose that the analysis of the 6 A data, especially the functional dependence of the EISF at $300 \mathrm{~K}$ and above, reflects the overlap of vibrational intensity with the quasielastic region. The limited momentum transfer $Q$ available in the $6 \AA$ measurements makes the analysis of the EISF and QISFs quite harsh and was therefore not attempted.

Hydrogen dynamics has been studied in several borohydride compounds in order to elucidate the connection with the crystal structure and dynamics. In alkali borohydrides, like $\mathrm{LiBH}_{4},{ }^{39,40}$ $\mathrm{NaBH}_{4}$ and $\mathrm{KBH}_{4},{ }^{41,42}$ the rotational reorientation is correlated with a crystal structure transition from an ordered (low-temperature) to a disordered (high-temperature) phase. In $\mathrm{NaBH}_{4}$ and $\mathrm{LiBH}_{4}$, hydrogen motions become faster as soon as the disordered state is reached. In the disordered phase of $\mathrm{KBH}_{4}$, the hydrogen reorientation (jump rotation around the $C_{4}$-fold axis) is similar to the one found in $\mathrm{NaBH}_{4}$. In $\mathrm{LiBH}_{4}$, the $\left[\mathrm{BH}_{4}\right]$ dynamics changes from an $C_{3}$ reorientation mechanism to a disordered reorientational model involving all four $\mathrm{H}$ atoms, as soon as the structural transition from orthorhombic (LT) to hexagonal (HT) is approached. ${ }^{43}$ Moreover, it has been suggested that the anomalous evolution of the lattice parameters as a function of temperature is associated with a pre-transition behaviour. ${ }^{44}$ Another mechanism in the crystal structure transition is found in $\mathrm{Ca}\left(\mathrm{BH}_{4}\right)_{2},{ }^{45}$ which is mainly driven by librations. Internal vibrations strongly affect the free energy and therefore drive the crystal phase transition.

In $\beta$ - $\mathrm{Mg}\left(\mathrm{BH}_{4}\right)_{2}$, we observe a dynamic transition between $200 \mathrm{~K}$ and $300 \mathrm{~K}$ : in the rotational part, at $300 \mathrm{~K}$ all $\left[\mathrm{BH}_{4}\right]$ units take part in the reorientation while only hindered rotations are observed at $200 \mathrm{~K}$; additionally, a transition from the underdamped to over-damped DHO is observed in that temperature range. The change in dynamics seems not to be connected to any structural phase transition. However, a recent study on $\beta-\mathrm{Mg}\left(\mathrm{BH}_{4}\right)_{2}{ }^{46}$ showed highly anisotropic, non-uniform thermal expansion of the lattice. In particular, the $b$-lattice parameter showed a maximum at around $275 \mathrm{~K}$, while for the $a$-lattice parameter a minimum value at approximately $200 \mathrm{~K}$ was observed. Moreover, the authors found that the refined atomic displacements did not detectably decrease with lower temperature. Our findings suggest a strong correlation between $\left[\mathrm{BH}_{4}\right]$ dynamics and the structural environment. The partial covalent character of $\beta-\mathrm{Mg}\left(\mathrm{BH}_{4}\right)_{2}$ appears to be connected with the crystal vibrational dynamics, in an intermediate position between ionic bonded borohydrides $\left(\mathrm{LiBH}_{4}\right.$ and $\left.\mathrm{NaBH}_{4}\right)$ to the more covalently bonded $\mathrm{Ca}\left(\mathrm{BH}_{4}\right)_{2}$.

\section{Conclusions}

In this work, QENS experiments have been performed on $\beta$ $\mathrm{Mg}\left(\mathrm{BH}_{4}\right)_{2}$ probing two different time-scales in order to explore the hydrogen dynamics, and in particular possible reorientations of the $\left[\mathrm{BH}_{4}\right]$ units. At longer timescales (up to $16 \mathrm{ps}, 6 \AA$ ), two different rotation motions have been detected. The slower one has an apparent activation energy of $32 \pm 3 \mathrm{meV}$. The second reorientation motion is also assigned to a rotational reorientation around $C_{2 \|}$ or $C_{2 \perp}$, however its temperature dependence is influenced by the underlying vibrations as it became obvious from the investigations at faster observation times. Below $300 \mathrm{~K}$, a distinct DHO signal appears in the measured data whereas at $300 \mathrm{~K}$ and above, it overlaps with the quasielastic signal. A transition from an under-damped to an over-damped regime is plausible. This transition in the scattering intensity strongly affects the EISF, QISF $_{\mathrm{QE}}$ and QISF $_{\text {inel }}$ and the vibrational and rotational motions could only be separated using a global fitting approach. From the experimental results, on both timescales, no long range diffusion of the $\left[\mathrm{BH}_{4}\right]$ units has been detected. In conclusion, the whole hydrogen dynamics in the picosecond timescale is dominated by both local rotational and vibrational reorientations occurring on similar timescales. 


\section{Appendix A}

The experimental data were analysed by a combination of rotational and vibrational reorientations (see eqn (3)). The scattering function $S_{\text {rot }}(Q, \omega)$ for jump rotational processes is described as the sum of a $\delta$-function (representing the elastic line) and a series of Lorentzian functions $L_{n}\left(\Gamma_{n}, \omega\right)$ :

$$
S_{\mathrm{rot}}(Q, \omega)=A_{0}(Q) \delta(\omega)+\sum_{n>0} A_{n}(Q) L_{n}\left(\Gamma_{n}, \omega\right)
$$

where the half-width at half-maximum (HWHM) $\Gamma_{n}$ are dispersionless and related to the medium residence time $\tau_{n}$ between successive jumps and $\sum_{n>0} A_{n}(Q)=1-A_{0}(Q)$. The time of the jump is assumed to be instantaneous. In the case of hindered rotations, that is a fraction of atoms $(p)$ not taking part in the reorientation, eqn (11) becomes:

$$
\begin{aligned}
S_{\mathrm{rot}}(Q, \omega)= & {\left[p+(1-p) A_{0}(Q)\right] \delta(\omega) } \\
& +(1-p) \sum_{n>0} A_{n}(Q) L_{n}\left(\Gamma_{n}, \omega\right),
\end{aligned}
$$

where the elastic term and quasielastic terms are again separated.

In the harmonic approximation, the vibrational scattering function $S_{\mathrm{vib}}(Q, \omega)$ can be written in a general form as the sum of elastic and inelastic terms:

$$
S_{\mathrm{vib}}(Q, \omega)=B(Q) \delta(\omega)+[1-B(Q)] S_{\mathrm{vib}}^{\mathrm{inel}}(Q, \omega),
$$

where $B(Q)$ is related to the mean square displacement of the nuclei, the Debye-Waller factor. The inelastic contribution $S_{\text {vib }}^{\text {inel }}(Q, \omega)$ was modeled using a damped harmonic oscillator ${ }^{47}(\mathrm{DHO})$ :

$$
S_{\mathrm{vib}}^{\mathrm{inel}}(Q, \omega)=\frac{n(\omega, T) Z(Q)}{\pi} \frac{4 \omega \Gamma_{q}}{\left[\left(\omega-\omega_{q}\right)^{2}+\Gamma_{q}^{2}\right]\left[\left(\omega+\omega_{q}\right)^{2}+\Gamma_{q}^{2}\right]}
$$

where $\omega_{q}$ is the renormalized vibration frequency and $\Gamma_{q}$ is the damping factor. The pre-factors $n(\omega, T)=\left(\mathrm{e}^{\hbar \omega / k_{\mathrm{B}} T}-1\right)^{-1}$ and $Z(Q)$ are, respectively, the Bose factor and DHO strength. The DHO is a double peak function around $\pm \omega_{q}$, in which the damping $\Gamma_{q}$ represents the broadening of the peaks; in the limit of zero damping, the DHO reduces to a double delta-function centred at $\pm \omega_{q} . \Gamma_{q}$ represents the decay constant, and its dependence on the energy and momentum transfer is in general not known. ${ }^{48}$ Two damping regimes can be distinguished: under-damped with $\Gamma_{q}<\omega_{q}$, and over-damped with $\Gamma_{q} \geq \omega_{q}$. In the latter case, the function assumes a single peak shape, centred at $\omega_{q}=0$.

The prefactor $B(Q)$ (Debye-Waller factor) generally depends on the phonon density of states and it is related to the mean square displacement of the atoms, ${ }^{48}$ in this case hydrogen. In the case of harmonic vibrations and only one type of scatterer, a general expression is given by

$$
B(Q)=\exp \left[-\left\langle(\vec{Q} \cdot \vec{u})^{2}\right\rangle\right]=\exp \left(-\frac{Q^{2}\left\langle u^{2}\right\rangle}{3}\right)
$$

where $u$ is the atomic displacement and the last inequality has been obtained by calculating the powder average denoted by brackets $\langle\cdots\rangle$. The expression for $B(Q)$ is strictly true in the harmonic approximation, although it has been observed that it is valid also in the presence of anharmonic effects. ${ }^{49}$

For purely rotational reorientations of the tetrahedra $\left[\mathrm{BH}_{4}\right]$ in $\mathrm{Mg}\left(\mathrm{BH}_{4}\right)_{2}$, around the $C_{2}$ or $C_{3}$ symmetry axis (see Fig. 2), the EISF (identical to $A_{0}$ ) is given by (in the powder average):

$$
A_{0}(Q)_{C_{2 / 3}}=\frac{1}{2}\left[1+j_{0}\left(\frac{2 \sqrt{2}}{\sqrt{3}} Q r\right)\right]
$$

where $j_{0}(x)=\sin (x) / x$ is the zeroth-order spherical Bessel function and $r=d_{\mathrm{B}-\mathrm{H}}$ is the bond length between boron and hydrogen atoms. For tumbling motions, among the hydrogen atoms, the EISF is:

$$
A_{0}(Q)_{\mathrm{tumb}}=\frac{1}{4}\left[1+3 j_{0}\left(\frac{2 \sqrt{2}}{\sqrt{3}} Q r\right)\right] .
$$

General expressions for $A_{0}$ can be found in the literature, e.g. for isotropic reorientation of the $\left[\mathrm{BH}_{4}\right]$ tetrahedra. ${ }^{37}$

Following the general expression of eqn (3), in the case of a single reorientation process (eqn (12) with $n=1$ ), and no translational $S_{\text {trans }}, S(Q, \omega)$ becomes:

$$
\begin{aligned}
S(Q, \omega)= & B\left[p+(1-p) A_{0}\right] \delta(\omega)+B(1-p) A_{1} L(\omega) \\
& +\left[p+(1-p) A_{0}\right](1-B) S_{\text {vib }}^{\text {inel }}(\omega) \\
& +(1-B)(1-p) A_{1}\left[S_{\text {vib }}^{\text {inel }}(\omega) \otimes L(\omega)\right],
\end{aligned}
$$

where the explicit dependence of $Q$ has been omitted for simplicity. Since all the scattering functions are normalized, the factors in front of them represent, respectively, the EISF, $\mathrm{QISF}_{\mathrm{QE}}, \mathrm{QISF}_{S_{\mathrm{vib}}}$ and $\mathrm{QISF}_{S_{\mathrm{vib}} \otimes L}$. These quantities can be evaluated from the data analysis. During the fit procedure, we found that the spectra can well be descibed by the sum of the elastic contribution, a quasielastic single Lorenzian and the vibrational contribution (for details see Table 2). The last two terms of eqn (18) could not be separated experimentally, and thus were summed to yield the following:

$$
\begin{gathered}
\mathrm{EISF}=B(Q)\left[p+(1-p) A_{0}(Q)\right] \\
\mathrm{QISF}_{\mathrm{QE}}=B(Q)(1-p)\left[1-A_{0}(Q)\right] \\
\mathrm{QISF}_{\text {inel }}=1-B(Q) .
\end{gathered}
$$

\section{Acknowledgements}

L. S. thanks Joachim Wuttke (Forschungszentrum Jülich (JCNS) and Heinz Maier-Leibnitz Zentrum (MLZ)) for the helpful discussion.

\section{References}

1 Y. Nakamori, K. Miwa, A. Ninomiya, H. Li, N. Ohba, S. Towata, A. Züttel and S. Orimo, Phys. Rev. B: Condens. Matter Mater. Phys., 2006, 74, 045126.

2 K. Chłopek, C. Frommen, A. Léon, O. Zabara and M. Fichtner, J. Mater. Chem., 2007, 17, 3496-3503. 
3 H.-W. Li, K. Kikuchi, Y. Nakamori, N. Ohba, K. Miwa, S. Towata and S. Orimo, Acta Mater., 2008, 56, 1342-1347.

4 T. Matsunaga, F. Buchter, P. Mauron, M. Bielman, Y. Nakamori, S. Orimo, N. Ohba, K. Miwa, S. Towata and A. Züttel, J. Alloys Compd., 2008, 459, 583-588.

5 G. L. Soloveichik, Y. Gao, J. Rijssenbeek, M. Andrus, S. Kniajanski, R. C. Bowman Jr., S.-J. Hwang and J.-C. Zhao, Int. J. Hydrogen Energy, 2009, 34, 916-928.

6 N. Hanada, K. Chlopek, C. Frommen, W. Lohstroh and M. Fichtner, J. Mater. Chem., 2008, 18, 2611-2614.

7 G. Severa, E. Rönnebro and C. M. Jensen, Chem. Commun., 2010, 46, 421-423.

8 M. Paskevicius, M. P. Pitt, C. J. Webb, D. A. Sheppard, U. Filsø, E. M. Gray and C. E. Buckley, J. Phys. Chem. C, 2012, 116, 15231-15240.

9 Y. Filinchuk, B. Richter, T. R. Jensen, V. Dmitriev, D. Chernyshov and H. Hagemann, Angew. Chem., Int. Ed., 2011, 50, 11162-11166.

10 W. I. F. David, S. K. Callear, M. O. Jones, P. C. Aeberhard, S. D. Culligan, A. H. Pohl, S. R. Johnson, K. R. Ryan, J. E. Parker, P. P. Edwards, C. J. Nuttall and A. AmieiroFonseca, Phys. Chem. Chem. Phys., 2012, 14, 11800-11807.

11 Y. Filinchuck, R. Černý and H. Hagemann, Chem. Mater, 2009, 21, 925-933.

12 J.-H. Her, P. W. Stephens, Y. Gao, G. L. Soloveichik, J. Rijssenbeek, M. Andrus and J.-C. Zhao, Acta Crystallogr., Sect. B: Struct. Sci., 2007, 63, 561-568.

13 R. Černý, Y. Filinchuk, H. Hagemann and K. Yvon, Angew. Chem., Int. Ed., 2007, 46, 5765-5767.

14 J. Yang, X. Zhang, J. Zheng, P. Song and X. Li, Scr. Mater., 2011, 64, 225-228.

15 Z. Zhang, H. Wang, J. Liu and M. Zhu, Thermochim. Acta, 2013, 560, 82-88.

16 R. J. Newhouse, V. Stavila, S.-J. Hwang, L. E. Klebanoff and J. Z. Zhang, J. Phys. Chem. C, 2010, 114, 5224-5232.

17 A. Al-Kukhun, H. T. Hwang and A. Varma, Int. J. Hydrogen Energy, 2012, 37, 17671-17677.

18 E. G. Bardají, Z. Zhao-Karger, N. Boucharat, A. Nale, M. J. van Setten, W. Lohstroh, E. Röhm, M. Catti and M. Fichtner, J. Phys. Chem. C, 2011, 115, 6095-6101.

19 B. Dai, D. S. Sholl and J. K. Johnson, J. Phys. Chem. C, 2008, 112, 4391-4395.

20 A. Bil, B. Kolb, R. Atkinson, D. G. Pettifor, T. Thonhauser and A. N. Kolmogorov, Phys. Rev. B: Condens. Matter Mater. Phys., 2011, 83, 224103.

21 R. Caputo, A. Kupczak, W. Sikora and A. Tekin, Phys. Chem. Chem. Phys., 2013, 15, 1471-1480.

22 V. Ozolins, E. H. Majzoub and C. Wolverton, Phys. Rev. Lett., 2008, 100, 135501.

23 J. Voss, J. S. Hummelshøj, Z. Łoodziana and T. Vegge, J. Phys.: Condens. Matter, 2009, 21, 012203.

24 X.-F. Zhou, A. R. Oganov, G.-R. Qian and Q. Zhu, Phys. Rev. Lett., 2012, 109, 245503.

25 Y. Filinchuk, D. Chernyshov and R. Černý, J. Phys. Chem. C, 2008, 112, 10579-10584.
26 W. H. Stockmayer and C. C. Stephenson, J. Chem. Phys., 1953, 21, 1311-1312.

27 G. Renaudin, S. Gomes, H. Hagemann, L. Keller and K. Yvon, J. Alloys Compd., 2004, 375, 98-106.

28 A. Borgschulte, R. Gremaud, A. Züttel, P. Martelli, A. Remhof, A. J. Ramirez-Cuesta, K. Refson, E. G. Bardají, W. Lohstroh, M. Fichtner, H. Hagemann and M. Ernst, Phys. Rev. B: Condens. Matter Mater. Phys., 2011, 83, 024102.

29 A. V. Skripov, A. V. Soloninin, O. A. Babanova, H. Hagemann and Y. Filinchuk, J. Phys. Chem. C, 2010, 114, 12370-12374.

30 A. V. Soloninin, O. A. Babanova, A. V. Skripov, H. Hagemann, B. Richter, T. R. Jensen and Y. Filinchuk, J. Phys. Chem. C, 2012, 116, 4913-4920.

31 D. Blanchard, J. B. Maronsson, M. D. Riktor, J. Kheres, D. Sveinbjørnsson, E. G. Bardají, A. Léon, F. Juranyi, J. Wuttke, K. Lefmann, B. C. Hauback, M. Fichtner and T. Vegge, J. Phys. Chem. C, 2012, 116, 2013-2023.

32 K. Chłopek, C. Frommen, A. Léon, O. Zabara and M. Fichtner, J. Mater. Chem., 2007, 17, 3496-3503.

33 T. Unruh, J. Neuhaus and W. Petry, Nucl. Instrum. Methods Phys. Res., Sect. A, 2007, 580, 1414-1422.

$34 \mathrm{~W}$. Lohstroh and Z. Evenson, Journal of large-scale research facilities JLSRF, 2015, 1, 1-3.

35 S. Cusack and W. Doster, Biophys. J., 1990, 58, 243-251.

36 R. Hempelmann, Quasielastic Neutron Scattering and Solid State Diffusion, Clarendon Press, 2000.

37 M. Bée, Quasielastic Neutron Scattering, Adam Hilger, 1988.

38 A. Giannasi, D. Colognesi, L. Ulivi, M. Zoppi, A. J. RamirezCuesta, E. G. Bardají, E. Röhm and M. Fichtner, J. Phys. Chem. A, 2010, 114, 2788-2793.

39 A. V. Skripov, A. V. Soloninin, Y. Filinchuk and D. Chernyshov, J. Phys. Chem. C, 2008, 112, 18701-18705.

40 N. Verdal, T. J. Udovic and J. J. Rush, J. Phys. Chem. C, 2012, 116, 1614-1618.

41 O. A. Babanova, A. V. Soloninin, A. P. Stepanov, A. V. Skripov and Y. Filinchuk, J. Phys. Chem. C, 2010, 114, 3712-3718.

42 N. Verdal, M. R. Hartman, T. Jenkins, D. J. DeVries, J. J. Rush and T. J. Udovic, J. Phys. Chem. C, 2010, 114, 10027-10033.

43 N. Verdal, T. J. Udovic, J. J. Rush, H. Wu and A. V. Skripov, J. Phys. Chem. C, 2013, 117, 12010-12018.

44 H. Hagemann, Y. Filinchuk, D. Chernyshov and W. van Beek, Phase Transitions, 2009, 82, 344-355.

45 D. Blanchard, M. D. Riktor, J. B. Maronsson, H. S. Jacobsen, J. Kehres, D. Sveinbjörnsson, E. G. Bardají, A. Léon, F. Juranyi, J. Wuttke, B. C. Hauback, M. Fichtner and T. Vegge, J. Phys. Chem. C, 2010, 114, 20249-20257.

46 Y. Filinchuk, R. Černý and H. Hagemann, Chem. Mater., 2009, 21, 925-933.

47 B. Fåk and B. Dorner, Phys. B, 1997, 234, 1107-1108.

48 S. Lovesey, Theory of Neutron Scattering from Condensed Matter, Clarendon Press, 1986.

49 A. A. Maradudin and P. A. Flinn, Phys. Rev., 1963, 129, 2529-2547. 